\title{
REVIEW
}

\section{Technical review of thyroid cancer effects by radiation of NPPs in Korea}

\author{
Kyo-Youn Kim ${ }^{\mathrm{a}}$, Keon Wook Kang ${ }^{\mathrm{b}}$, Dongkwon Keumc, Bonghwan Kim ${ }^{\mathrm{a}}$, Kwang Pyo Kimc, Byung Il Kimd, \\ Songwon Seo ${ }^{\mathrm{d}}$, Young-Woo Jin ${ }^{\mathrm{d}}$, Soo-Geun Kim ${ }^{\mathrm{e}}$, Hyun Jung Kim ${ }^{\mathrm{f}}$, Hyeong Sik Ahn ${ }^{\mathrm{f}}$, Kunwoo Cho ${ }^{\mathrm{g}}$, Kyuhwan Jeong ${ }^{\mathrm{g}}$ \\ and Woo-Yoon Park ${ }^{\mathrm{h}}$ \\ ${ }^{a}$ Korea Atomic Energy Research Institute(KAERI), Daejeon, Korea; ${ }^{b}$ Seoul National University, Seoul, Korea; ${ }^{c}$ KyungHee University, \\ Suwon, Korea; ${ }^{d}$ Korea Institute of Radiological \& Medical Science(KIRAMS), Seoul, Korea; ${ }^{e}$ Sungkyunkwan University, Seoul, \\ Korea; ${ }^{f}$ Korea University, Seoul, Korea; ${ }^{g}$ Korea Institute of Nuclear Safety(KINS), Daejeon, Korea $;{ }^{h}$ Chungbuk National \\ University, Cheongju, Korea
}

\begin{abstract}
After the Fukushima accident and Busan district's court ruling partially in favor of some of the plaintiffs, the residents near Nuclear Power Plants (NPPs) have suffered from ungrounded fears. To obliterate their fear and anxiety, the correlation between the occurrence of thyroid cancer and radiation from NPPs has been analyzed by experts in this paper. At a dose range of $100 \mathrm{mSv}$ or below, this study suggests that there is no scientific evidence that carcinogenesis increases based on the radiation dose. In conclusion, no causal relation has been shown between NPPs and the occurrence of thyroid cancer of residents living near NPPs. Government supported research should be increased to relieve ungrounded anxiety in the future.
\end{abstract}

Keywords: radiation; cancer; thyroid cancer; epidemiology; nuclear power plants; residents

\section{Introduction}

After Japan was hit by the Fukushima Daiichi nuclear power plant accident caused by an earthquake and following a tsunami in Fukushima on March 11, 2011, public scrutiny on nuclear power and radiation has heightened. However, along with the skyrocketing interests in radiation followed by the Fukushima nuclear disaster, distorted information has been circulated in the media such as newspapers and TV news and social networks and received by the public non-discriminately, thereby creating hysteria on radiation. However, the radiation dose around nuclear power plant (NPP) remains lower than one-tenth of the natural background radiation in Korea. Amid these circumstances, three residents living near the Kori NPP filed damage claims against Korea Hydro \& Nuclear Power (KNHP) on July 4, 2012. On October 17, 2014, the Eastern Branch of the Busan District Court ruled partially in favor of the plaintiffs on the first trial that the defendant should compensate one of the plaintiffs (a wife) for her thyroid cancer at 15,000,000 won (about 14,000USD), although it dismissed the claims of the two other plaintiffs, a husband for his rectal cancer, and a son for his congenital autism.

The essence of the ruling of the first trial court is that "the plaintiffs have been exposed to the release of radioactive materials from the nearby NPP after having lived in that area for an extended period of time, which

*Corresponding author. Email: sky@kaeri.re.kr provides the significant reason for the diagnosis of cancer, and therefore, this decision holds the defendant accountable for compensation."

Although the radiation dose in the region around the NPPs remains at the same level as the natural background radiation of any other region in Korea, thanks to the safe management of radiation of NPPs, the decision of the original trial has made nearby inhibiting residents feel unsettled without any clear evidence to support their fears.

To settle this unfounded public anxiety, the Korean Nuclear Society (KNS) and Korean Association for Radiation Protection (KARP) decided to create an independent committee served by experts from the academic and research communities and set out to make a clear and fact-based diagnosis on the correlations between the occurrence of thyroid cancer and radiation in the surrounding areas of NPPs.

\section{Material and methods}

Domestic and international studies on the relationship between radiation and adverse health effects, especially solid cancers and thyroid cancer, were examined and reviewed. For instance, a Life Span Study (LSS) on the atomic bomb survivors in Japan, epidemiological studies on the residents exposed to radiation from the Chernobyl accident, measurements of radiation in Korea and foreign countries, and other many studies and data were reviewed by the committee. The committee then had 
several meetings and discussions to compare and analyze the existing researches and decided to publish this technical note to relieve groundless fears of the public for radiation and NPPs by providing a scientific analysis and conclusions of the committee.

\section{Results and discussion}

An epidemiology study on NPPs by the Medical Research Center of Seoul National University, which provided the basis for the claim of relevance between NPPs and thyroid cancer, concluded that there has been no clear relationship between NPPs and the incidence of cancer in residents living near NPPs. However, in the case of women, it showed a meaningful increase in the incidence of thyroid cancer incidence in proportion to the decrease in the distance between residence and NPPs, and thus some pessimists deduced that radiation affects the incidence of thyroid cancer. However, if this reasoning is logical, not only the incidence of thyroid cancer but other types of cancer (e.g., breast cancer) should have increased. In addition, the incidence rate of thyroid cancer should have risen in both men and women but it was observed that the incidence rate increased dramatically only in women.

Moreover, the incidence rate of thyroid cancer was not proportionate to the period of living near NPPs according to an epidemiological survey [1]. Table 1 shows the incident rate of thyroid cancer in women depending on the distance from a nuclear power plant. It may seem there is relevance between the distance from a nuclear power plant and the incidence of thyroid cancer because the value of relative risk in the surrounding area is 2.5 , which is statistically valid. However, in the short distance region, the value of relative risk is statistically invalid and even lower than that of the surrounding area. If there is a tendency of increase in the incidence of thyroid cancer when the distance from the nuclear power plant becomes shorter, the correlation of all the data should be consistent, but they are not. In this case, living near a nuclear power plant cannot be suggested as a reason for the incidence of thyroid cancer.

Table 1. Incident rate of thyroid cancer in women by epidemiology study for NPP in Korea [1].

\begin{tabular}{cccc}
\hline & \multirow{2}{*}{$\begin{array}{c}\text { Surrounding } \\
\text { area (within } \\
\text { Index }\end{array}$} & \multicolumn{2}{c}{ Control region } \\
\cline { 3 - 4 } & $\begin{array}{c}\text { Short } \\
\text { distance } \\
(5 \sim 30 \mathrm{~km})\end{array}$ & $\begin{array}{c}\text { Long } \\
\text { distance } \\
\text { (over 30km) }\end{array}$ \\
\hline $\begin{array}{c}\text { Incidence } \\
\text { rate }\end{array}$ & 61.4 & 43.6 & 26.6 \\
\hline $\begin{array}{c}\text { Relative } \\
\text { risk } \\
(95 \% \mathrm{CI})\end{array}$ & 2.5 & $\begin{array}{c}1.8 \\
(1.43-4.38)\end{array}$ & 1.0 \\
\hline
\end{tabular}

Table 2 shows that the radiation dose of residents living near NPPs annually also supports this point. The highest dose that the residents receive per year is approximately $0.1 \mathrm{mSv} /$ year. This is the same as one-tenth of the annual dose limit for the public by ICRP [2], and $4 \%$ of the average background radiation of the world [3]. In addition, the relative risk of nuclear workers working within $0.5 \mathrm{~km}$ of an NPP is 1.06 , which is lower than that of residents. Therefore, the incidence of cancer in relation to the proximity from NPPs shows inconsistent results as a whole. The opinion that the incidence of thyroid cancer is strongly related to the radiation dose from NPPs cannot have significant meaning.

Table 2. Radiation dose of residents living near NPP [4].

\begin{tabular}{ccccc} 
& & \multicolumn{3}{c}{ Unit: $\mathrm{mSv} / \mathrm{yr}$} \\
\cline { 2 - 5 } Yaer & \multicolumn{3}{c}{ Nuclear Power Plants } \\
\cline { 2 - 5 } & Kori & Wolsong & Hanbit & Hanul \\
\hline 2004 & 0.00520 & 0.00460 & 0.00600 & 0.00240 \\
2005 & 0.00512 & 0.00285 & 0.00301 & 0.00338 \\
2006 & 0.00664 & 0.00348 & 0.00485 & 0.00165 \\
2007 & 0.01510 & 0.00579 & 0.00604 & 0.00209 \\
2008 & 0.00460 & 0.00831 & 0.00957 & 0.00190 \\
2009 & 0.00226 & 0.00154 & 0.00432 & 0.00207 \\
2010 & 0.00152 & 0.00520 & 0.00273 & 0.00333 \\
2011 & 0.00171 & 0.00481 & 0.00271 & 0.00330 \\
2012 & 0.00418 & 0.02231 & 0.01612 & 0.01568 \\
2013 & 0.00455 & 0.02859 & 0.00584 & 0.01205 \\
2014 & 0.00268 & 0.10520 & 0.00801 & 0.02610 \\
\hline
\end{tabular}

According to real-time and nation-wide surveillance data on environmental radiation (http://iernet.kins.re.kr), the radiation dose near NPPs is managed at around 0.01 $\mathrm{mSv}$ or below, and the dose rates in the surrounding areas of NPPs do not show any significant differences from other regions where NPPs are not located. Therefore, although some residents might have lived near NPPs over 20 years, it has not been clearly identified that they had been exposed to more radiation compared to residents of other regions. Instead, insisting that the natural background radiation is a cause of the incidence of cancer might be more logical. On average, it is estimated that Koreans receive $3.08 \mathrm{mSv}$ annually, and radon makes up around $50 \%$ of this amount [5]. However, the radon level shows differences depending on region, and even the lifestyle of individuals affects the level of natural background radiation.

The average level of indoor radon concentration of various regions in Korea. The level of indoor radon concentration varies with the composition of rocks and soil and the building materials [6]. Comparing the nationwide average, radon concentrations in the North Jeolla Province and the South Chungcheong Province appear to be fairly high, and as a result, the annual effective dose rate is expected to be around $1.7 \mathrm{mSv}$. The radon concentration levels for Ulsan and Busan are roughly $1 \mathrm{mSv}$, which is slightly lower than the national average. The citizens in Ulsan who receive $0.01 \mathrm{mSv}$ of radon annually are exposed to much less radon than the 
residents living in the North Jeolla Province who receive $0.7 \mathrm{mSv}$, and thus the residents living near NPPs are considered to be exposed to the smallest amount of radon in Korea. The average indoor radon concentration of Korea is $54 \mathrm{~Bq} / \mathrm{m} 3$, which is lower than the recommended annual average concentration reference level of radon, $100 \mathrm{~Bq} / \mathrm{m} 3$ [7]. Even compared with the world's indoor radon concentration, shown in Figure 1, although there are some differences of indoor radon concentration among countries, Korea's indoor radon concentration is almost the same with that of the average concentration of other countries.

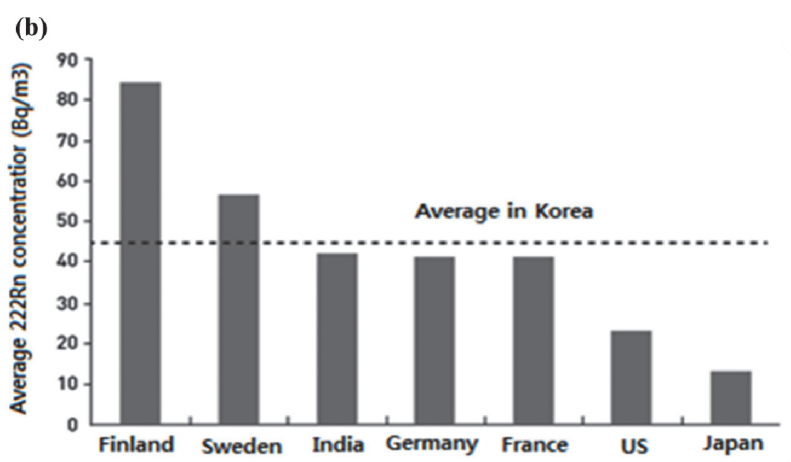

Figure 1. Accumulated incidence rate of thyroid cancer by gender and radiation dose of surrounding areas of NPP [5].

No epidemiology studies on residents living near normally operating NPPs have reported an increase in the incidence of thyroid cancer. A retrospective cohort study was conducted to track whether people exposed to Iodine-131 released from nuclear power facilities in Hanford, US when they were children, have an increased tendency of thyroid cancer. From December 1992 to September 1997, a health examination was carried out on the exposure cases taken place from 1944 to 1957. An analysis was performed on the relationship between the radiation dose and medical check-up results of 3,440 people among the total of 5,199 subjects. As a result, an analysis discovered no relevance between the radiation dose in Hanford and thyroid-related diseases (thyroid cancer, thyroid nodule, autoimmune thyroiditis, and hypothyroidism) [8]. Figure 2 indicates the accumulated incidence rate of thyroid cancer by gender and radiation dose of surrounding areas of NPPs in Hanford, US. However, the accumulated incidence rate of thyroid cancer of both men and women and all ages near the NPPs in Hanford is lower than $1 \%$. In addition, the incidence rate of thyroid cancer of these areas is lower than that of outside regions.

An ecological study on whether residents living in the surrounding areas of NPPs in Belgium have an increased number of incidence of thyroid cancer also showed no increase in the incidence of thyroid cancer in proportion to proximity to nuclear power plants [10]. In addition to this, one study on the cancer incidence and mortality of residents living near NPPs in Borgo, Italy reported no statistically meaningful observation of cancer incidence in both men and women including thyroid cancer [11]. Korea adopted the strictest standard of ICRP (ICRP-60) prior to advanced countries, even prior to the US. It is reasonable to consider that the risk of residents living near NPPs in Korea is lower than or at least the same with that of other countries that operate NPPs.

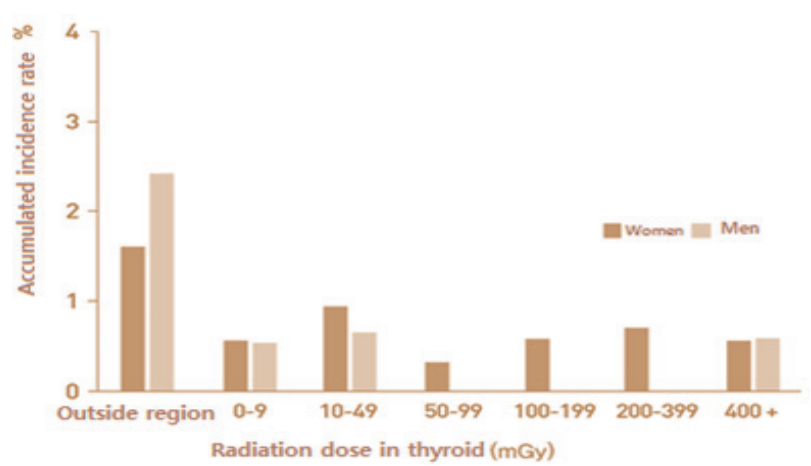

Figure 2. Accumulated incidence rate of thyroid cancer by gender and radiation dose of surrounding areas of NPPs in Hanford, US [9].

According to the research on Hiroshima and Nagasaki atomic bomb survivors and the study on residents living near NPPs in Chernobyl, there has been no scientific evidence that the increase in the number of the incidences of thyroid cancer is related to radiation exposure among adults aged 20 or more. However, the incidence of thyroid cancer caused by radiation exposure was collectively observed in a pediatric age group who ate radioiodine-polluted vegetables, milk, etc. [12,13]. Since the Chernobyl nuclear accident, a health impact study on local residents has been continuously carried out. The most apparent and well-known health impact discovered thus far is the incidence of thyroid cancer in children. At the time of the accident, among 116,131 residents, the average thyroid absorbed dose was estimated to be $1.82 \mathrm{~Gy}$ for children aged 7 , and under and 0.29 Gy for adults. The estimated absorbed dose of residents living in nearby regions (Belarus, Ukraine, Pripyat) was $0.97-3.1 \mathrm{~Gy}$ for children aged 7 and under, and $0.07-0.68 \mathrm{~Gy}$ for adults. The most common route to radiation exposure is by drinking milk polluted with Iodine-131.

Every study on analyzing the impact of Chernobyl disaster has consistently reported the relevance between the incidence of pediatric thyroid cancer and radiation [9]. A study targeted for a group of people aged 18 and under conducted in Belarus and Ukraine found that the statistically meaningful excess relative risk (ERR) of thyroid cancer was 8 to 19 during the observational period, from 1990 to 2001. In Belarus, from 1991 to $2005,6,848$ children and teenagers aged 18 or less developed thyroid cancer. In Figure 3, in Belarus, the pattern of incidence of thyroid cancer among children under age 10 years, from 1991 to 1995, shows the correlation of thyroid cancer with the Chernobyl accident. However, for those who born after 1986, there is no special relationship between the incidence of thyroid cancer and the accident. In addition, for adults, no relevance between radiation exposure and the development of thyroid cancer has been observed [14,15]. 


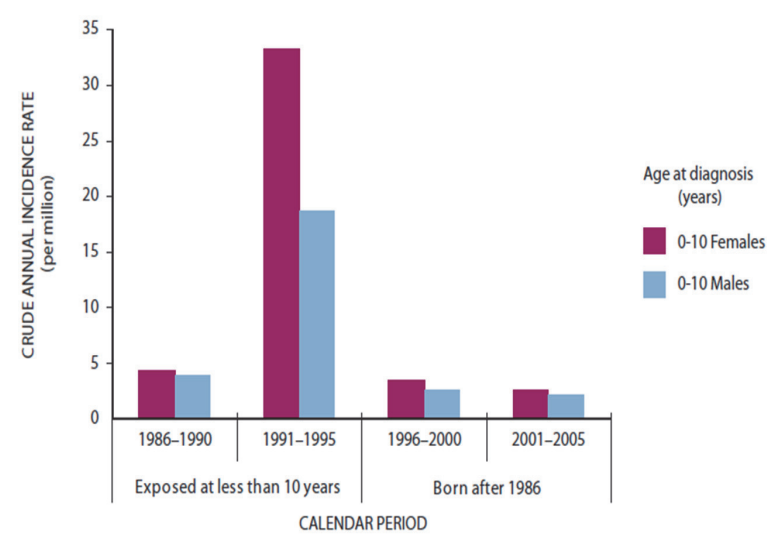

Figure 3. Incidence of pediatric thyroid cancer in Belarus [16].

The advancement in diagnostic technology of thyroid cancer and the popularization of medical check-ups have been cited as the main cause of the increase in the incidence of thyroid cancer in women. Many women without jobs were not able to benefit from health screenings at work, but recently they have received the benefits of national health screening and expanded coverage. In 1999, the government launched a national cancer screening project and other medical examinations as a national project and provided a discount for medical check-ups on breast cancer, colorectal cancer, stomach cancer, and liver cancer, or offered services free of charge through this project. In addition, the residents living near NPPs have more opportunities of receiving medical screening for thyroid cancer compared to other local residents, thanks to the medical benefits offered by nuclear power plants and the local government. Nevertheless, the diagnosis rate of thyroid cancer $(1.3 \%)$ of residents living near NPPs in Gijang is not higher than that of a large hospital in Seoul $(2.5 \%)$ where no relevance with nuclear power plants exists [15].

According to the national cancer registration, as shown in Figure 4, the incidence of thyroid cancer per 100,000 women is 134.1 in 2011 , which is the highest in the world [17].

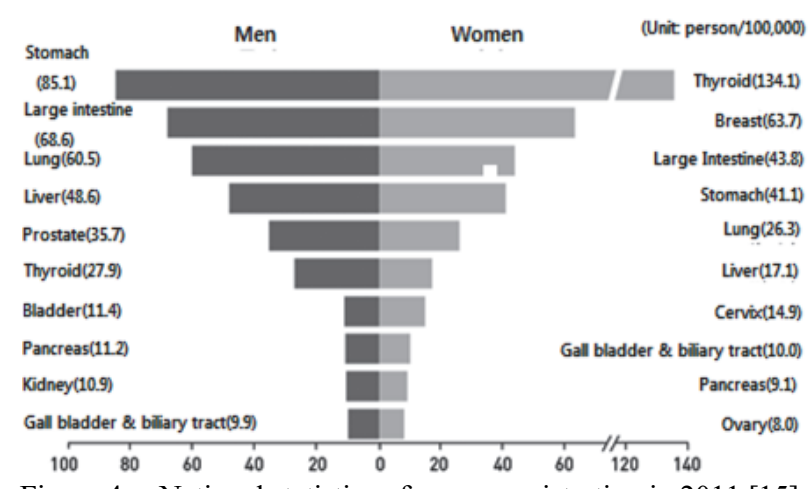

Figure 4. National statistics of cancer registration in 2011 [15].

As shown in Figure 5, the incidence of thyroid cancer slightly increased in the 1990s and showed dramatic increases after 2000. In 2011, the diagnosis of thyroid cancer increased 15 times compared to that observed in 1993. In particular, the most dramatic increase was observed in papillary carcinoma. The mortality caused by thyroid cancer maintains the same level with the past although the incidence of thyroid cancer has exponentially increased. The fatality rate of thyroid cancer for 15 years from 1993 to 2011 was almost not changed at 0.7 persons per 100,000 , in spite of the consistent increase in thyroid cancer, which provides the basis for supporting the claim of the increased detection of thyroid cancer. In other words, though the number of patients increased through early screening, mortality did not change much because thyroid cancer was not a life-threatening cancer.

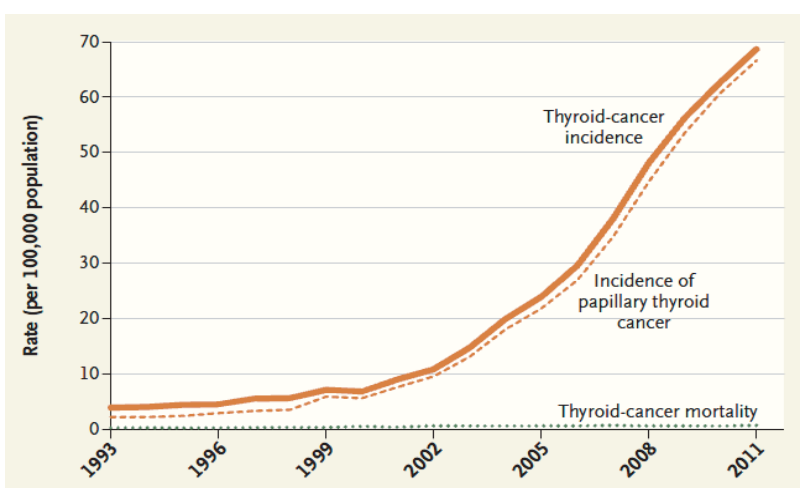

Figure 5. Progress of incidence rate of thyroid cancer and papillary thyroid carcinoma and fatality rate from thyroid cancer in Korea (1993-2011) [18].

As shown in Figure 6, the incidence of thyroid cancer differed in 16 provinces, and it was explained by the rate of screening of thyroid cancer per region. In 2010, the Korean Community Health Survey (KCHS), researching roughly 200,000 people nationwide, was conducted for adults aged 19 and above to inquire whether they received a thyroid cancer screening test within the past two years. The connection between the incidence rate of thyroid cancer per region in 2008 and 2009 was derived from the National Cancer Registration Program data. The implementation rate of thyroid cancer screening collected from KCHS was analyzed based on region, age group, and gender, which presented a stronger relevance than other data $[16,17]$.

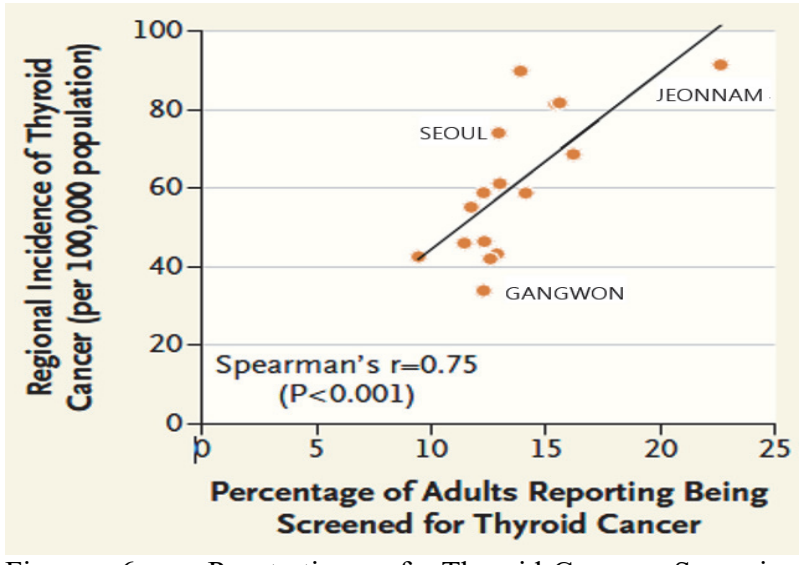

Figure 6. Penetration of Thyroid-Cancer Screening (2008-2009) and Incidence of Thyroid Cancer (2009) in the 16 Administrative Regions of South Korea [18]. 
With the data mentioned above, an increase in thyroid cancer was concluded by the rise of the diagnostic prevalence rate given the fact that medical technology, including fine-needle aspiration biopsy using ultrasound, has been widely accessible, and that the incidence rate of mainly micro-carcinoma has increased, and that micro-carcinoma has been increasingly discovered through medical examination screening for other diseases, and that thyroid cancer with no symptoms, later confirmed through an autopsy, has risen [19]. In particular, the dramatic increase of thyroid cancer in Korea coincides with a trend of the increasing medical check-ups, including the rising accessibility of ultrasound screening tests. Therefore, taking into account the impacts of the increased examination is the most critical factor in analyzing the trend of rising thyroid cancer and the causes behind it.

As shown in Figure 5, the incidence rate of thyroid cancer was higher in areas with early screening rates. The early screening rate of thyroid cancer in Jeonnam province was the highest in Korea at $22.63 \%$, and the incidence rate of thyroid cancer in Jeonnam province was 91.8 people per 100,000 populations. The early screening rate of thyroid cancer in Gangwon province was the lowest in Korea at $12.28 \%$, and the incidence rate of thyroid cancer in Gangwon province was 37.1 people per 100,000 people. In other words, early regional screening of thyroid cancer has a high correlation with the increase in thyroid cancer incidence in Korea [20].

\section{Conclusion}

When radiation biology and the effects of radiation on humans were not well known, people did not care when they were treated with radiation, and were exposed to high doses. Many studies have focused on such people, Japanese atomic bomb survivors, or workers who have suffered from high doses of radiation owing to accidents of nuclear power plants to suggest radiation as a reason for cancer. However, a vast amount of knowledge has been accumulated through historical experience and studies for more than 100 years, and thus people can now handle radiation safely, and radiation has become necessary in the diagnosis and treatment of diseases, and in many other fields.

As stated above, cancer is the number one reason of death for Koreans. Moreover, recently, the dramatic increase of thyroid cancer in Koreans has triggered both domestic and international interest. There are many suggested causes of thyroid cancer, but it is believed that many factors complexly affect its occurrence. Therefore, it is quite difficult to clearly elucidate the cause of individual thyroid cancer.

Until now, there has been no certain method to distinguish naturally occurring thyroid cancer from radiation-causing thyroid cancer. Thus, to prove that the tendency of the recent thyroid cancer rate is caused by radiation, there should be scientific evidence that exposure only to radiation affects thyroid cancer, and a comparative study and a follow-up study should be conducted at a large scale for a long period of time.

In addition, as mentioned above, there is no causal relationship between normally operating nuclear power plants and the occurrence of thyroid cancer of residents living near NPPs. Radiation emitted from NPPs is strictly regulated under international recommendations. In addition, more frequent medical checkups increase the possibility of detecting thyroid cancer. However, there is ungrounded mistrust regarding radiation because information and publications on the relationship to the public are lacking or too difficult for non-specialists. The government and relevant organizations and groups should double their efforts to relieve the ungrounded mistrust on radiation considering such non-specialists. Further, the government should increase its support of research into what kind of impact radiation has on the human body to allay idle fears regarding radiation and to deliver exact information about radiation to the public.

The following conclusions were made through this study:

1) An epidemiology study on NPPs by Seoul National University showed no causation between NPPs and thyroid cancer, even though some inconsistent data were shown in the report.

2) Residents near NPPs are exposed to $0.01 \mathrm{mSv}$ or below from plants, which is an extremely small value compared to radiation from cosmic rays and natural radionuclides.

3) No epidemiological studies on residents living near NPPs in foreign countries have reported an increase in thyroid cancer cases.

4) Research on the Hiroshima atomic bomb survivors and residents living near Chernobyl NPPs has shown no evidence of increased cancer incidence in adults.

5) An increase in the number of medical exams on thyroid cancer is the main cause for the increased rates of thyroid cancer in women.

\section{Acknowledgements}

The authors wish to thank Korean Nuclear Society and Korean Association for Radiation Protection for the support of this study.

\section{References}

[1] Y.O. Ahn, Epidemiological investigation on cancer risk among radiation workers in nuclear power plants and residents nearby nuclear power plant in Korea, MEST (2011).

[2] ICRP, ICRP Publication 103 (2007).

[3] CNSC, Fact Sheet - Natural Background Radiation (2013).

[4] KHNP, Study and Report on Environmental Radiation near NPPs (2004 - 2013).

[5] KINS, Radiation environment in Korea, KINS/GR-356, (2009).

[6] J.A. Gunby, Factors affecting indoor radon 
concentrations in the United Kingdom, Health Physics Society 64 (1993), pp.2-12.

[7] WHO, Radon and Health - Fact Sheet (2016).

[8] National Academies, Exposure of the American People to Iodine-131 from Nevada Nuclear-Bomb Tests (1999).

[9] CDC, HTDS Guide (2010).

$[10] \mathrm{K}$. Bollaerts, Thyroid cancer incidence in the vicinity of nuclear site in Belgium, Thyroid 5 (2014), pp.906-917.

[11]F. Mataloni, Cancer incidence and mortality in the cohort of residents close to the Italian Nuclear Power Plants of Borgo Sabotino and Garigliano, Epidemiol Prev. 5 (2012), pp.253-262.

[12]E. Cardis, The chernobyl accident - an epidemiological perspective, Clin. Oncol. 4 (2011), pp.251-260.

[13]K. Ozasa, Studies of the mortality of atomic bomb survivors, Report 14, 1950-2003: An overview of cancer and noncancer diseases, Radiation Research 177 (2012), pp.229-243.
[14]P. Jacob, Thyroid cancer risk in areas of Ukraine and Belarus affected by the chernobyl, Radiat. Res. 1 (2006), pp.1-8.

[15]I. Likhtarov, Post-chernobyl thyroid cancers in Ukraine. Report 2: Risk analysis, Radiat. Res. 2 (2006), pp.375-386.

[16] UNSCEAR, UNSCEAR 2008 - Health Effects due to Radiation from Chernobyl Accident (2008).

[17]Ministry of Health and Welfare, Statistics of National Cancer Registration in 2011 (2013).

[18]H.S. Ahn, H.J. Kim and H.G. Welch, Korea's thyroid-cancer "Epidemic" screening and overdiagnosis, N. Engl. J. Med. 19 (2014), pp.1765-1767.

[19]J.H. Lee, Overdiagnosis and screening for thyroid cancer in Korea, The Lancet, 9957 (2014), p.1848.

[20]H.S. Ahn and H.J. Kim, Correlation of regional screening, incidence rate, and mortality rate of thyroid cancer, 2015 Spring Workshop, KNS 2015 Conference, Jeju, Korea (2015). 